\title{
Light-Assisted Growth of Hexagonal Au Nanostructures on Sapphire Substrates
}

\author{
Spencer D. Golze ${ }^{1}$, Robert D. Neal ${ }^{1}$, Robert A. Hughes ${ }^{1}$, Svetlana Neretina ${ }^{1}$ and Sergei Rouvimov ${ }^{2}$ \\ 1. Department of Aerospace and Mechanical Engineering, University of Notre Dame, Notre Dame, IN. \\ 2. Department of Electrical Engineering, University of Notre Dame, Notre Dame, IN.
}

Noble metal nanostructures currently have a wide range of applications in the fields of chemistry, catalysis, electronics, bio-science, and medicine [1]. For example, Au nanoparticles can be used for the detection of biological and chemical analytes using various sensing modalities (e.g., Surface Enhanced Raman Scattering (SERS)). The fabrication of homogeneous arrays of metal nanoparticles of the desired size, shape, and composition is of importance in achieving and maintaining nanostructures with certain physical properties. In addition to light-mediated syntheses [1] which allows for the production of a monodisperse population of nanoparticles in solution, homogenous and periodically distributed arrays of hexagonal metallic nanoplates can also be fabricated using a room temperature light-activated growth mode performed on seeds immobilized on a crystalline substrate [2]. The latter synthesis is reliant on the formation of $\mathrm{Au}$ seeds exhibiting planar defects, whereby the desired growth mode is deactivated without such defects.

Here, the growth mechanisms and defect formation in Au nanostructures have been studied based on SEM and TEM analysis. A combination of High Resolution TEM and STEM imaging as well as Selected Area Electron Diffraction performed by FEI Titan 80-300 Transmission Electron Microscope has been employed for structural and compositional characterization of the samples. TEM crosssectional samples were prepared using an FEI Helios SEM/FIB dual beam tool. Figure 1a shows a typical SEM image of a periodic array of hexagonal $\mathrm{Au}$ nanoplates formed on a (0001)-oriented sapphire substrate. The cross-sectional TEM images (Fig. 1,b and c) provide evidence that the hexagonal $\mathrm{Au}$ nanoplates contain planar defects (twins, and stacking faults) that are parallel to the substrate surface. The presence of twins in the nanoplates is also evidenced by the corresponding electron diffraction pattern (Fig. 1d).

The orientation relationship between the Au nanoplates and sapphire substrate is dictated by an epitaxial relationship given by $(111)_{\mathrm{Au}} / /(0001)_{\mathrm{sapph}},(110)_{\mathrm{Au}} / /(1-100)_{\mathrm{sapph}}$. It is, however, interesting that there is only a small area where the nanoplate is epitaxially connected to the substrate while the majority of the nanoplate was formed by a lateral overgrowth mechanism. The formation of stacking faults and microtwins during growth may be associated with strain near the $\mathrm{Au}-\mathrm{Al}_{2} \mathrm{O}_{3}$ interface due to high $(\sim 4-5 \%)$ lattice mismatch between $\mathrm{Au}$ and sapphire. The lateral overgrowth allows for the minimization of strain in the periodic assembly of Au nanoplates. The ADF STEM image shown in Fig. 2 evidences the presence of twins in Au crystalline seeds. The HRTEM image of a Au seed (Fig. 3) illustrates that the shape of $\mathrm{Au}$ nanostructures is directly related to the location of the twin defect and the orientations of (111) planes. The latter may define the facets of the Au nanoplates. The mechanisms of defect formation and their relation to the nanoplate growth mode will be discussed [3]. 


\section{References:}

[1] M. R. Langille, M. L. Personick, and C. A. Mirkin, Angew. Chem. Int. Ed. 52 (2013), p. 13910.

[2] Y. Zhai et al, Nature Materials 15 (2016), p. 889.

[3] The authors acknowledge the support of this study by NDIIF and a National Science Foundation Award (DMR-1707593) to S. N.
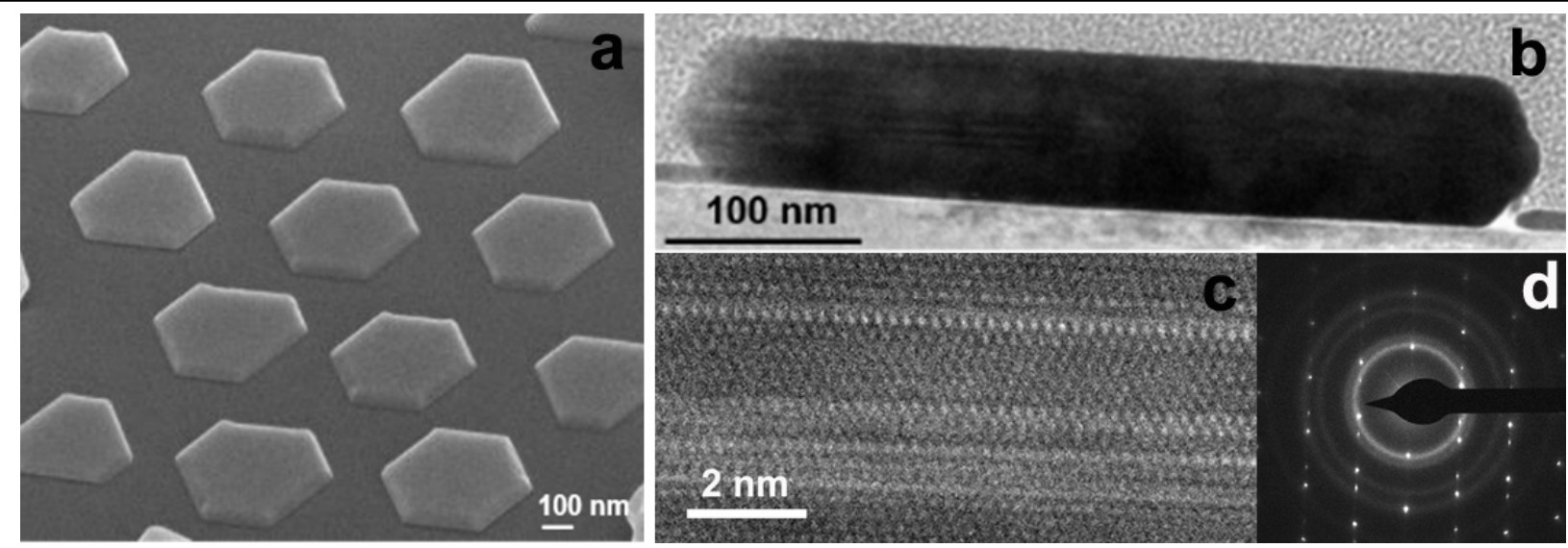

Figure 1. SEM (a) and Cross-sectional TEM (b) images of hexagonal Au NPs that contains twins and stacking faults. The presence of twins in the nanostructure image in (b) is also evidenced by corresponding electron diffraction pattern (d).

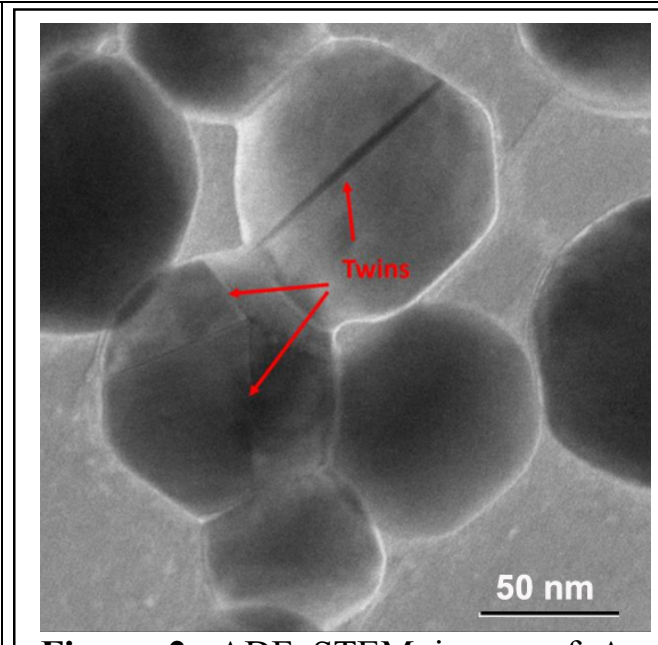

Figure 2. ADF STEM image of $\mathrm{Au}$ nanoparticle seeds that exhibit twin structures.

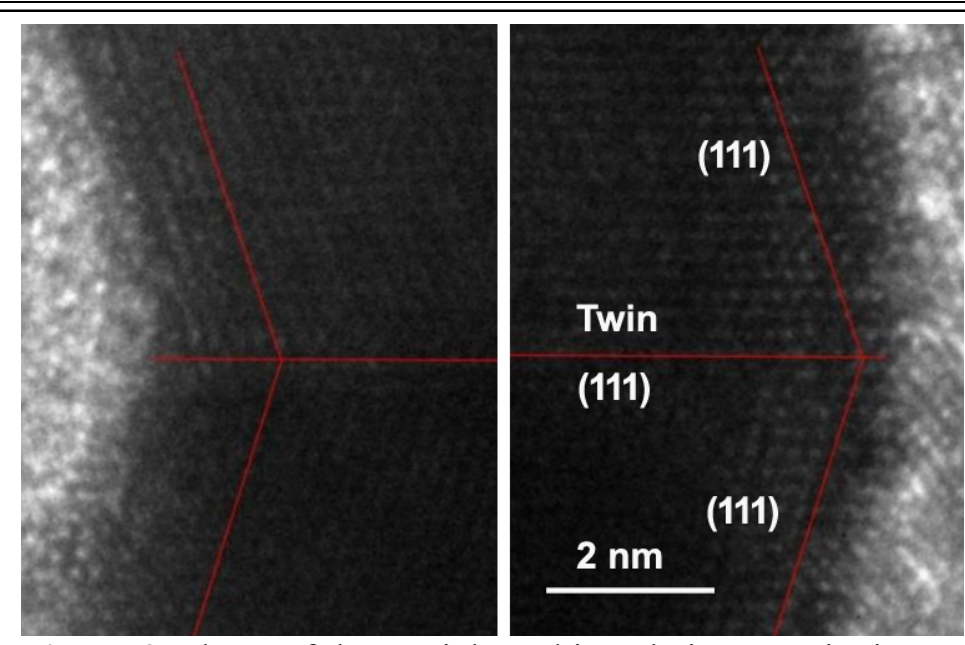

Figure 3. Shape of the particle and its relation to twinning as observed in an HRTEM image. 